Journal of Systems Science and Information

Dec., 2016, Vol. 4, No. 6, pp. 547-559

DOI: $10.21078 /$ JSSI-2016-547-13

\title{
Analysis of a Single-Sever Queue with Disasters and Repairs Under Bernoulli Vacation Schedule
}

\author{
Jingjing YE \\ School of Science, Nanjing University of Science and Technology, Nanjing 210094, China \\ E-mail: 568657332@qq.com \\ Liwei LIU \\ School of Science, Nanjing University of Science and Technology, Nanjing 210094, China \\ E-mail: lwliu@mail.njust.edu.cn \\ Tao JIANG \\ School of Science, Nanjing University of Science and Technology, Nanjing 210094, China \\ E-mail: jtao0728@163.com
}

\begin{abstract}
This paper studies a single-sever queue with disasters and repairs, in which after each service completion the server may take a vacation with probability $q(0 \leqslant q \leqslant 1)$, or begin to serve the next customer, if any, with probability $p(=1-q)$. The disaster only affects the system when the server is in operation, and once it occurs, all customers present are eliminated from the system. We obtain the stationary probability generating functions (PGFs) of the number of customers in the system by solving the balance equations of the system. Some performance measures such as the mean system length, the probability that the server is in different states, the rate at which disasters occur and the rate of initiations of busy period are determined. We also derive the sojourn time distribution and the mean sojourn time. In addition, some numerical examples are presented to show the effect of the parameters on the mean system length.
\end{abstract}

Keywords Bernoulli vacation schedule; disasters; strong Markov property; sojourn time

\section{Introduction}

With the development of communication systems and networks, queueing systems with disasters have been paid more and more attention. In recent twenty years, the topic on the queue with disasters has been largely studied by many researchers. Once a total disaster occurs, all customers present (including the one in service) have to leave the system. This kind of queueing systems can be applied in many fields. For example, when an ATM in a bank is out of order, all customers have to leave. Also, in computer networks, a virus as a clearing operation can remove all stored datum present.

Since the introduction of disasters and their characters, there has been considerable attention paid to this topic. Kumar and Arivudainambi ${ }^{[1]}$ studied the transient solution of an 
$\mathrm{M} / \mathrm{M} / 1$ queue with catastrophes and derived the transient solution for the system size and some performance measures. In their paper, whenever a catastrophe occurred, all the customers present were destroyed, the server turned to idle and waited for a new arrival. Yang and $\mathrm{Kim}^{[2]}$ studied an M/G/1 stochastic clearing system, and obtained the system size distribution and the sojourn time distribution. Among some classical papers on disasters, readers may refer to Economou and Fakinos ${ }^{[3]}$, Gani and Swift ${ }^{[4]}$, Shin ${ }^{[5]}$. Recently, Yechiali ${ }^{[6]}$ studied the queue with system disasters and impatient customers in which when the system is in breakdown, the new arrival starts an impatience time. In this paper, he derived some important service measures, such as the mean sojourn time of a served customer, the rate of lost customers due to disasters and the proportion of customers served. Other excellent papers on the model with impatient customers have been reported by Sudhesh ${ }^{[1]}$, Dimou and Economou ${ }^{[12]}$. Jolai and Asadzadeh ${ }^{[7]}$ studied a finite source discrete time Geo/Geo/1 queue with disasters, Park and Yang ${ }^{[8]}$ extended it to the GI/Geo/1 queue. Economou and Gómez-Corral ${ }^{[15]}$ studied a batch Markovian arrival process subject to renewal generated geometric catastrophes. Boudali and Economou $^{[9]}$ studied optimal and equilibrium balking strategies in the single server Markovian queue with catastrophes and derived the corresponding Nash equilibrium and social optimal strategies. Later, they analyzed the effect of catastrophes on the strategic customer behavior in queueing systems ${ }^{[10]}$. Kim and Lee ${ }^{[13]}$ studied an $M / G / 1$ queue with disasters and working breakdowns. Yi and $\mathrm{Kim}^{[14]}$ studied a Geo/G/1 queue with disasters and multiple working vacations, and presented the steady-state queue length distribution. Jiang, et al. ${ }^{[17]}$ studied an $\mathrm{M} / \mathrm{G} / 1$ queue in a multi-phase random environment with disasters. By using the supplementary variable technique, they obtained the distribution for the stationary queue and derived the results of the cycle analysis, the sojourn time distribution and the length of working time in a service cycle. In the same year, they studied a GI/M/1 queue in a multi-phase service environment with disasters and working breakdowns in which instead of stopping service, the sever continued to serve the customer at a lower rate during the repair period ${ }^{[18]}$. After that, Zhang, et al. ${ }^{[20]}$ studied an $\mathrm{M} / \mathrm{G} / 1$ stochastic clearing queue in 3-phase environment.

During the recent twenty years, there are few articles about the queue with total disasters and vacations. Mytalas and Zazanis ${ }^{[16]}$ are the first as we know. They considered an $M^{X} / \mathrm{G} / 1$ queueing system with disasters and repairs under a multiple adapted vacation policy in which if there is no customer at the epoch of the busy period termination or at the end of a repair period in the system, the sever may take a string of vacations. In their paper, they obtained some rate arguments, the fraction of customers who complete service, and the Laplace Stieltjes Transform (LST) of the system time of a typical customer. Queue with sever vacations have been studied extensively since the late 70's, and readers may refer to the excellent paper which give a short survey on the recent developments in vacation queueing models ${ }^{[19]}$.

Bernoulli vacation schedule is also developed significantly due to its wide applications and flexibility. In daily life, while a sever completes a service, he is also possible to take a vacation for some reason even if there are customers in the system. For example, when a driver completes a transport business, he may choose to have a rest whether or not according to his own physical condition. Further, this kind of vacations not just a particular schedule, also provides the opportunity to analyze the same queue model with single vacation and no vacation. Motivated 
by above-mentioned, in this paper, we consider a single-sever queue with disasters and repairs under Bernoulli vacation schedule. Not only do we present the PGF of the number of customers in the system, but also obtain the sojourn time of a customer and some performance measures.

The rest of this paper is organized as follows. In Section 2, we present the description of the queue model. In Section 3, the steady-state queue size distribution are obtained by solving balance equations. In Section 4, we derive some performance measures and rate arguments. In Section 5 , the LST of the sojourn time distribution is determined. Some numerical examples are given to show the influence of parameters on the mean system length in Section 7. Section 8 is the conclusion.

\section{Model Description}

Consider an $\mathrm{M} / \mathrm{M} / 1$ queueing system with disasters and repairs under Bernoulli vacation schedule. Customers arrive to the system according to a Poisson process with rate $\lambda$ and are served singly under the first-come-first-serve (FCFS) service discipline. The service times are assumed to be independent, identically distributed (i.i.d.) random variables with common exponentially distributed with parameter $\mu$. Whenever the service of a customer is completed, the server may take a vacation with probability $q(0 \leqslant q \leqslant 1)$, or begin to serve the next customer, if any, with probability $p(=1-q)$. Further, it is assumed that the durations of vacations are i.i.d. random variable and follow exponentially distributed with rate $\eta$. During the busy periods, the system may suffer a disaster which will clear of all customers present (including customers being served and waiting in line) and lead to the system empty. Meanwhile the server initiates a repair period. Both the interarrival times of disasters and the repair times are exponentially distributed with parameter $\alpha$ and $r$, respectively. Finally, we assume that the arrival process, the service times, the durations of vacations and the repair times are mutually independent of each other.

\section{The Steady-State Queue Size Distribution}

In this section, we model a two-dimensional Markov chain which is denoted by $\{(I(t), N(t))$, $t \geqslant 0\}$ to describe the system state. At time $t, I(t)$ denotes the status of the server and $N(t)$ the number of customers in the system. The state space of the Markov process is $\Omega=$ $\{\{(3,0)\} \cup\{(1, k)\} \cup\{(i, n)\}, i=0,2, k=1,2, \cdots, n=0,1, \cdots\}$, where $(3,0)$ represents the server being idle, 1 and $i=0,2$ correspond to serving a customer, taking a vacation and being under repair. As we know, due to the occurrence of the disaster, all customers present are eliminated from the system. Therefore, as long as we set $\alpha>0$, the system in consideration is always stable.

We now define

$$
\begin{array}{ll}
\pi_{0, n}=\lim _{t \rightarrow \infty} P\{I(t)=0, N(t)=n\}, & n \geqslant 0, \\
\pi_{1, n}=\lim _{t \rightarrow \infty} P\{I(t)=1, N(t)=n\}, & n \geqslant 1, \\
\pi_{2, n}=\lim _{t \rightarrow \infty} P\{I(t)=2, N(t)=n\}, & n \geqslant 0, \\
\pi_{3,0}=\lim _{t \rightarrow \infty} P\{I(t)=3, N(t)=0\} . &
\end{array}
$$

These denote the stationary probabilities of the Markov chain, and satisfy the following 
balance equations

$$
\begin{aligned}
& (\lambda+\eta) \pi_{0,0}=\mu q \pi_{1,1}, \\
& (\lambda+\eta) \pi_{0, n}=\mu q \pi_{1, n+1}+\lambda \pi_{0, n-1}, \quad n \geqslant 1, \\
& \lambda \pi_{3,0}=\mu p \pi_{1,1}+\eta \pi_{0,0}+r \pi_{2,0}, \\
& (\lambda+\mu+\alpha) \pi_{1,1}=\mu p \pi_{1,2}+\eta \pi_{0,1}+r \pi_{2,1}+\lambda \pi_{3,0}, \\
& (\lambda+\mu+\alpha) \pi_{1, n}=\mu p \pi_{1, n+1}+\lambda \pi_{1, n-1}+\eta \pi_{0, n}+r \pi_{2, n}, \quad n \geqslant 2, \\
& (\lambda+r) \pi_{2,0}=\alpha \sum_{n=1}^{\infty} \pi_{1, n}, \\
& (\lambda+r) \pi_{2, n}=\lambda \pi_{2, n-1}, \quad n \geqslant 1 .
\end{aligned}
$$

The normalization condition is

$$
\pi_{3,0}+\sum_{n=0}^{\infty} \pi_{0, n}+\sum_{n=1}^{\infty} \pi_{1, n}+\sum_{n=0}^{\infty} \pi_{2, n}=1
$$

In order to obtain the steady-state probabilities, we define partial PGFs as follow

$$
G_{0}(z)=\sum_{n=0}^{\infty} \pi_{0, n} z^{n}, \quad G_{1}(z)=\sum_{n=1}^{\infty} \pi_{1, n} z^{n}, \quad G_{2}(z)=\sum_{n=0}^{\infty} \pi_{2, n} z^{n} .
$$

Then, we have

$$
\pi_{3,0}+G_{0}(1)+G_{1}(1)+G_{2}(1)=1
$$

Multiplying (1) and (2) by $1, z^{n}$, respectively, and summing over $n$, we have

$$
G_{0}(z)=\frac{\mu q G_{1}(z)}{\eta z+\lambda z(1-z)} .
$$

Multiplying (6) and (7) by $1, z^{n}$, respectively, and performing the summations over $n$, we have

$$
G_{2}(z)=\frac{\alpha G_{1}(1)}{r+\lambda(1-z)}
$$

From (3), (4) and (5), we obtain

$$
G_{1}(z)=\frac{\eta z G_{0}(z)+r z G_{2}(z)-\lambda z(1-z) \pi_{3,0}}{\alpha z+\mu(z-p)+\lambda z(1-z)} .
$$

Substituting (10) into (12), we have

$$
G_{1}(z)=\frac{r z G_{2}(z)-\lambda z(1-z) \pi_{3,0}}{\alpha z+\mu(z-p)+\lambda z(1-z)-\frac{\mu q \eta}{\eta+\lambda(1-z)}} .
$$

Theorem 1 The quantities $G_{1}(1)$ and $\pi_{3,0}$ satisfy the following relationship

$$
\frac{r \alpha G_{1}(1)}{r+\lambda\left(1-z^{*}\right)}=\lambda\left(1-z^{*}\right) \pi_{3,0},
$$

where $z^{*}$ is the unique root of the equation

$$
f(z)=\alpha z+\mu(z-p)+\lambda z(1-z)-\frac{\mu q \eta}{\eta+\lambda(1-z)}
$$

in $|z|<1$. 
Proof Now, let $z=0$ and $z=1$ in (15), respectively, we have

$$
f(0)=-\mu p-\frac{\mu q \eta}{\eta+\lambda}<0, \quad f(1)=\alpha>0 .
$$

When the function $f(z)$ has at least one root in $|z|<1$. Next, by taking the first and second derivative of function $f(z)$, we have

$$
\begin{aligned}
& f^{\prime}(z)=-2 \lambda z+(\alpha+\mu+\lambda)-\frac{\lambda \mu q \eta}{[\eta+\lambda(1-z)]^{2}}, \\
& f^{\prime \prime}(z)=-2 \lambda-\frac{2 \lambda^{2} \mu q \eta}{[\eta+\lambda(1-z)]^{3}} .
\end{aligned}
$$

Obviously, $f^{\prime \prime}(z)<0$ in $|z|<1$, so we get that $f^{\prime}(z)$ is a monotonous decreasing function in $|z|<1$. Let $z=0$, we have

$$
\begin{aligned}
& f^{\prime}(0)=(\alpha+\mu+\lambda)-\frac{\lambda \mu q \eta}{(\eta+\lambda)^{2}}, \\
& f^{\prime}(0)(\eta+\lambda)^{2}=(\alpha+\mu+\lambda)(\eta+\lambda)^{2}-\lambda \mu q \eta, \\
& f^{\prime}(0)(\eta+\lambda)^{2}=(\alpha+\mu p+\lambda)(\eta+\lambda)^{2}+\mu q\left(\eta^{2}+\lambda^{2}+\lambda \eta\right)>0 .
\end{aligned}
$$

Hence $f^{\prime}(0)>0$. Let $z=1$, we have

$$
\begin{aligned}
& f^{\prime}(1)=-2 \lambda+(\alpha+\mu+\lambda)-\frac{\lambda \mu q}{\eta}, \\
& f^{\prime}(1) \eta=(\alpha+\mu) \eta-\lambda(\mu q+\eta) .
\end{aligned}
$$

When $\lambda \leqslant \frac{(\alpha+\mu) \eta}{\mu q+\eta}, f^{\prime}(1) \geqslant 0$, by $f^{\prime}(0)>0$ and $f^{\prime \prime}(z)<0$, we have that $f(z)$ is a monotonous increasing function in $|z|<1$. From $f(0)<0, f(1)>0$, we obtain that $f(z)$ has a unique root in $|z|<1$.

When $\lambda>\frac{(\alpha+\mu) \eta}{\mu q+\eta}, f^{\prime}(1)<0$. from $f^{\prime}(0)>0$ and $f^{\prime \prime}(z)<0$, we note that $\exists k \in(0,1)$, so that $f^{\prime}(k)=0$, then we have that $f(z)$ is a monotonous increasing function in $(0, k)$ and a monotonous descreasing function in $(k, 1)$. From $f(0)<0, f(1)>0$, we have that $f(z)$ has a unique root in $|z|<1$.

In summary, $f(z)$ has a unique root in $|z|<1$. Due to the occurrence of the disaster, the system in consideration is always stable. Therefore, the power series $G_{1}(z)$ in (13) is converges in the unit circle $|z|<1$, i.e., $G_{1}(z)$ must be finite for all $|z|<1$. Let $z=z^{*}$ be the unique root of $f(z)$. In (13), as the denominator vanishes as $z \rightarrow z^{*}$, the numerator must vanish for the root as well. Then, Substituting (11) into the numerator, (14) follows.

From (9), (10), (11) and (14), we can obtain

$$
G_{1}(1)=\frac{\lambda \eta r\left(1-z^{*}\right)\left[r+\lambda\left(1-z^{*}\right)\right]}{\alpha \eta r^{2}+\lambda(\mu q r+\alpha \eta+\eta r)\left(1-z^{*}\right)\left[r+\lambda\left(1-z^{*}\right)\right]}
$$

and

$$
\pi_{3,0}=\frac{\lambda \eta \alpha r^{2}}{\alpha \eta r^{2}+\lambda(\mu q r+\alpha \eta+\eta r)\left(1-z^{*}\right)\left[r+\lambda\left(1-z^{*}\right)\right]} .
$$

Substituting (16) into (11), we have

$$
G_{2}(z)=\frac{\lambda \eta \alpha r\left(1-z^{*}\right)\left[r+\lambda\left(1-z^{*}\right)\right]}{[r+\lambda(1-z)]\left\{\alpha \eta r^{2}+\lambda(\mu q r+\alpha \eta+\eta r)\left(1-z^{*}\right)\left[r+\lambda\left(1-z^{*}\right)\right]\right\}} .
$$


Substituting (17) and (18) into (13), we have

$$
G_{1}(z)=\frac{\lambda \eta \alpha r^{2} z\left\{\left(1-z^{*}\right)\left[r+\lambda\left(1-z^{*}\right)\right]-\lambda(1-z)[r+\lambda(1-z)]\right\}}{f(z)[r+\lambda(1-z)]\left\{\alpha \eta r^{2}+\lambda(\mu q r+\alpha \eta+\eta r)\left(1-z^{*}\right)\left[r+\lambda\left(1-z^{*}\right)\right]\right\}} .
$$

From (10) and (19), we can obtain

$$
G_{0}(z)=\frac{\lambda \mu q \eta \alpha r^{2}\left\{\left(1-z^{*}\right)\left[r+\lambda\left(1-z^{*}\right)\right]-\lambda(1-z)[r+\lambda(1-z)]\right\}}{f(z)[\eta+\lambda(1-z)][r+\lambda(1-z)]\left\{\alpha \eta r^{2}+\lambda(\mu q r+\alpha \eta+\eta r)\left(1-z^{*}\right)\left[r+\lambda\left(1-z^{*}\right)\right]\right\}} .
$$

Theorem 2 The PGF of the number of customers in the system is

$$
G(z)=\pi_{3,0}+G_{0}(z)+G_{1}(z)+G_{2}(z)
$$

where $\pi_{3,0}, G_{0}(z), G_{1}(z)$ and $G_{2}(z)$ are given by (17), (20), (19) and (18), respectively.

\section{Performance Measures}

\subsection{The Mean Number of Customers in the Systems}

Let $L$ be the number of customers in the system, then

$$
\begin{aligned}
E[L]=G^{\prime}(1)= & G_{0}^{\prime}(1)+G_{1}^{\prime}(1)+G_{2}^{\prime}(1) \\
= & \frac{\lambda \mu q\left\{\lambda \alpha \eta r^{2}-\left(1-z^{*}\right)\left[r+\lambda\left(1-z^{*}\right)\right][(\alpha+\mu-\lambda) \eta r-\lambda \mu q r-\lambda \alpha(\eta+r)]\right\}}{\alpha \eta\left\{\alpha \eta r^{2}+\lambda(\mu q r+\alpha \eta+\eta r)\left(1-z^{*}\right)\left[r+\lambda\left(1-z^{*}\right)\right]\right\}} \\
& +\frac{\lambda\left\{\lambda \alpha \eta r^{2}+\left(1-z^{*}\right)\left[r+\lambda\left(1-z^{*}\right)\right](\lambda \eta r-\mu \eta r+\lambda \mu q r+\lambda \alpha \eta)\right\}}{\alpha\left\{\alpha \eta r^{2}+\lambda(\mu q r+\alpha \eta+\eta r)\left(1-z^{*}\right)\left[r+\lambda\left(1-z^{*}\right)\right]\right\}} \\
& +\frac{\lambda^{2} \alpha \eta\left(1-z^{*}\right)\left[r+\lambda\left(1-z^{*}\right)\right]}{r\left\{\alpha \eta r^{2}+\lambda(\mu q r+\alpha \eta+\eta r)\left(1-z^{*}\right)\left[r+\lambda\left(1-z^{*}\right)\right]\right\}} .
\end{aligned}
$$

\subsection{The Probability of the Server in Different States}

Let $P_{w}$ be the probability of the server in the busy periods, then

$$
P_{w}=G_{1}(1)=\frac{\lambda \eta r\left(1-z^{*}\right)\left[r+\lambda\left(1-z^{*}\right)\right]}{\alpha \eta r^{2}+\lambda(\mu q r+\alpha \eta+\eta r)\left(1-z^{*}\right)\left[r+\lambda\left(1-z^{*}\right)\right]} .
$$

Let $P_{r}$ be the probability of server in the repair periods, then

$$
P_{r}=G_{2}(1)=\frac{\lambda \eta \alpha\left(1-z^{*}\right)\left[r+\lambda\left(1-z^{*}\right)\right]}{\alpha \eta r^{2}+\lambda(\mu q r+\alpha \eta+\eta r)\left(1-z^{*}\right)\left[r+\lambda\left(1-z^{*}\right)\right]} .
$$

Let $P_{v}$ be the probability that the sever is in a vacation, then

$$
P_{v}=G_{0}(1)=\frac{\lambda \mu q r\left(1-z^{*}\right)\left[r+\lambda\left(1-z^{*}\right)\right]}{\alpha \eta r^{2}+\lambda(\mu q r+\alpha \eta+\eta r)\left(1-z^{*}\right)\left[r+\lambda\left(1-z^{*}\right)\right]} .
$$

And $\pi_{3,0}$ is the probability that the sever is idle.

\subsection{Rate Arguments}

As we know, disasters occur and affect the system only when the server is serving customers. Therefore, the rate at which disasters occur, $R_{d i s}$, is

$$
R_{d i s}=\alpha G_{1}(1)=\frac{\lambda \eta \alpha r\left(1-z^{*}\right)\left[r+\lambda\left(1-z^{*}\right)\right]}{\alpha \eta r^{2}+\lambda(\mu q r+\alpha \eta+\eta r)\left(1-z^{*}\right)\left[r+\lambda\left(1-z^{*}\right)\right]} .
$$


$R_{d i s}$ is also the rate of busy period terminations due to disasters.

Next, let us determine the rate of busy period terminations due to finish serving all customers present. Obviously, the rate $R_{\text {ser }}$ is

$$
R_{\text {ser }}=\mu \pi_{1,1}=\frac{1}{p}\left(\lambda \pi_{3,0}-\eta \pi_{0,0}-r \pi_{2,0}\right)
$$

where, in the above equation, we used (3). Let $z=0$ in (18) and (20), we can obtain

$$
\begin{aligned}
\pi_{2,0} & =\frac{\lambda \eta \alpha r\left(1-z^{*}\right)\left[r+\lambda\left(1-z^{*}\right)\right]}{(r+\lambda)\left\{\alpha \eta r^{2}+\lambda(\mu q r+\alpha \eta+\eta r)\left(1-z^{*}\right)\left[r+\lambda\left(1-z^{*}\right)\right]\right\}}, \\
\pi_{0,0} & =\frac{\lambda q \eta \alpha r^{2}\left\{\left(1-z^{*}\right)\left[r+\lambda\left(1-z^{*}\right)\right]-\lambda(r+\lambda)\right\}}{(-\eta-\lambda p)(r+\lambda)\left\{\alpha \eta r^{2}+\lambda(\mu q r+\alpha \eta+\eta r)\left(1-z^{*}\right)\left[r+\lambda\left(1-z^{*}\right)\right]\right\}} .
\end{aligned}
$$

Then substitute (17), (28) and (29) into (27), we have

$$
R_{\text {ser }}=\frac{\lambda \eta \alpha r^{2}(\lambda+\eta)\left\{\lambda(r+\lambda)-\left(1-z^{*}\right)\left[r+\lambda\left(1-z^{*}\right)\right]\right\}}{(\eta+\lambda p)(r+\lambda)\left\{\alpha \eta r^{2}+\lambda(\mu q r+\alpha \eta+\eta r)\left(1-z^{*}\right)\left[r+\lambda\left(1-z^{*}\right)\right]\right\}} .
$$

As we know, each busy period initiation always follows a previous one termination due to either the occurrence of a disaster or the service completion. Hence, the rate of initiations of busy periods, $R_{\text {bus }}$, is given by

$$
\begin{aligned}
R_{\text {bus }}= & R_{\text {dis }}+R_{\text {ser }} \\
= & \frac{\lambda \eta \alpha r\left(1-z^{*}\right)\left[r+\lambda\left(1-z^{*}\right)\right]}{\alpha \eta r^{2}+\lambda(\mu q r+\alpha \eta+\eta r)\left(1-z^{*}\right)\left[r+\lambda\left(1-z^{*}\right)\right]} \\
& +\frac{\lambda \eta \alpha r^{2}(\lambda+\eta)\left\{\lambda(r+\lambda)-\left(1-z^{*}\right)\left[r+\lambda\left(1-z^{*}\right)\right]\right\}}{(\eta+\lambda p)(r+\lambda)\left\{\alpha \eta r^{2}+\lambda(\mu q r+\alpha \eta+\eta r)\left(1-z^{*}\right)\left[r+\lambda\left(1-z^{*}\right)\right]\right\}} .
\end{aligned}
$$

Theorem 3 The PGF of the number of all customers present at initiation epoch of a busy period is given by

$$
\varphi(z)=\frac{1}{R_{\text {bus }}}\left[\lambda \pi_{3,0} z+\eta\left(G_{0}(z)-\pi_{0,0}\right)+r\left(G_{2}(z)-\pi_{2,0}\right)\right],
$$

where $\pi_{3,0}, G_{0}(z), \pi_{0,0}, G_{2}(z), \pi_{2,0}$ and $R_{\text {bus }}$ are given by (17), (20), (29), (18), (28) and (31), respectively.

Proof The rate of busy period initiations that start with $n$ customers present is denoted by $R_{n}$. Obviously,

$$
R_{n}=\delta_{n, 1} \lambda \pi_{3,0}+\eta \pi_{0, n}+r \pi_{2, n}, \quad n \geqslant 1 .
$$

Therefore, the ratio of busy periods initiations that start with $n$ customers present is

$$
\varphi_{n}=\frac{R_{n}}{R_{\text {bus }}}, \quad n \geqslant 1 .
$$

The PGF of the number of all customers present at initiation epoch of a busy period is

$$
\varphi(z)=\sum_{n=1}^{\infty} \frac{R_{n}}{R_{b u s}} z^{n} .
$$

Substituting (31) and (33) into (35), (32) follows. 


\section{The LST of the Stationary Sojourn Time Distribution of an Arbi- trary Customer}

Considering a tagged customer who is the $(n+1)$ th customer standing in line, i.e., at the instant of the tagged customer arriving at the system, there have just been $n$ customers in the system (including the one being served). The sojourn time for a tagged customer begins from the moment that he enters the system and ends the moment that he leaves the system due to either the occurrence of a disaster or the service completion. Here, let $W$ denotes the stationary sojourn time of an arbitrary customer and $W^{*}(s)$ corresponds to its LST.

Theorem 4 The LST of the stationary sojourn time distribution of an arbitrary customer is given by

$$
\begin{aligned}
W^{*}(s)= & \pi_{3,0} \frac{\mu+\alpha}{\mu+\alpha+s}+\sum_{n=0}^{\infty} \pi_{0, n} \frac{\eta}{\eta+s}\left(A^{n} \frac{\mu+\alpha}{\mu+\alpha+s}+\frac{B\left(1-A^{n}\right)}{1-A}\right) \\
& +\sum_{n=1}^{\infty} \pi_{1, n}\left(A^{n} \frac{\mu+\alpha}{\mu+\alpha+s}+\frac{B\left(1-A^{n}\right)}{1-A}\right) \\
& +\sum_{n=0}^{\infty} \pi_{2, n} \frac{r}{r+s}\left(A^{n} \frac{\mu+\alpha}{\mu+\alpha+s}+\frac{B\left(1-A^{n}\right)}{1-A}\right),
\end{aligned}
$$

where $A=\frac{\mu \eta+\mu p s}{(\mu+\alpha+s)(\eta+s)}, B=\frac{\alpha}{\mu+\alpha+s}$.

Proof Now, consider several different states in which the tagged customer arrives and derive the LST of the stationary sojourn time.

The first one is $(3,0)$. When the tagged customer arrives, the server is idle and he/she is the only one in the system. Hence, the server starts to serve the tagged customer immediately, and he/she departs by either the occurrence of a disaster or the service completion. Letting $W_{3,0}^{*}(s)$ denote the LST of the stationary sojourn time of the tagged customer in this case, we have

$$
W_{3,0}^{*}(s)=E\left(\mathrm{e}^{-s \min (X, \Delta)}\right)=\frac{\mu+\alpha}{\mu+\alpha+s},
$$

where $X$ and $\Delta$ represent the interarrival of customers and disasters, respectively.

The second one is $(0, n), n=0,1, \cdots$. When the tagged customer arrives, the server is taking a vacation and there are $n$ customers in front of him/her. Let $W_{0, n}^{*}(s), n=0,1, \cdots$, denote the LST of the stationary sojourn time of the tagged customer in this case. By using strong Markov property, we can obtain

$$
\begin{aligned}
& W_{0,0}^{*}(s)=\frac{\lambda}{\lambda+\eta+s} W_{0,0}^{*}(s)+\frac{\eta}{\lambda+\eta+s} W_{3,0}^{*}(s), \\
& W_{0, n}^{*}(s)=\frac{\lambda}{\lambda+\eta+s} W_{0, n}^{*}(s)+\frac{\eta}{\lambda+\eta+s} W_{1, n}^{*}(s), \quad n \geqslant 1 .
\end{aligned}
$$

The third one is $(1, n), n=1,2, \cdots$. When the tagged customer arrives, the server is serving a customer and there are $n$ customers in front of him/her. Let $W_{1, n}^{*}(s), n=1,2, \cdots$, denote the LST of the stationary sojourn time of the tagged customer in this case. By using strong 
Markov property, we have

$$
\begin{aligned}
W_{1,1}^{*}(s)= & \frac{\lambda}{\lambda+\mu+\alpha+s} W_{1,1}^{*}(s)+\frac{\mu p}{\lambda+\mu+\alpha+s} W_{3,0}^{*}(s) \\
& +\frac{\mu q}{\lambda+\mu+\alpha+s} W_{0,0}^{*}(s)+\frac{\alpha}{\lambda+\mu+\alpha+s} \cdot 1, \\
W_{1, n}^{*}(s)= & \frac{\lambda}{\lambda+\mu+\alpha+s} W_{1, n}^{*}(s)+\frac{\mu p}{\lambda+\mu+\alpha+s} W_{1, n-1}^{*}(s) \\
& +\frac{\mu q}{\lambda+\mu+\alpha+s} W_{0, n-1}^{*}(s)+\frac{\alpha}{\lambda+\mu+\alpha+s} \cdot 1, \quad n \geqslant 2 .
\end{aligned}
$$

The fourth one is $(2, n), n=0,1, \cdots$. When the tagged customer arrives, the system is under repair and there are $n$ customers in front of him/her. Let $W_{2, n}^{*}(s), n=0,1, \cdots$, denote the LST of the stationary sojourn time of the tagged customer in this case. By using strong Markov property, we can obtain

$$
\begin{aligned}
& W_{2,0}^{*}(s)=\frac{\lambda}{\lambda+r+s} W_{2,0}^{*}(s)+\frac{r}{\lambda+r+s} W_{3,0}^{*}(s), \\
& W_{2, n}^{*}(s)=\frac{\lambda}{\lambda+r+s} W_{2, n}^{*}(s)+\frac{r}{\lambda+r+s} W_{1, n}^{*}(s), \quad n \geqslant 1 .
\end{aligned}
$$

Solving (38) (43), we have

$$
\begin{aligned}
W_{0,0}^{*}(s) & =\frac{\eta}{\eta+s} W_{3,0}^{*}(s), \\
W_{0, n}^{*}(s) & =\frac{\eta}{\eta+s} W_{1, n}^{*}(s), \quad n \geqslant 1, \\
W_{1,1}^{*}(s) & =\frac{\mu p}{\mu+\alpha+s} W_{3,0}^{*}(s)+\frac{\mu q}{\mu+\alpha+s} W_{0,0}^{*}(s)+\frac{\alpha}{\mu+\alpha+s}, \\
W_{1, n}^{*}(s) & =\frac{\mu p}{\mu+\alpha+s} W_{1, n-1}^{*}(s)+\frac{\mu q}{\mu+\alpha+s} W_{0, n-1}^{*}(s)+\frac{\alpha}{\mu+\alpha+s}, \quad n \geqslant 2, \\
W_{2,0}^{*}(s) & =\frac{r}{r+s} W_{3,0}^{*}(s), \\
W_{2, n}^{*}(s) & =\frac{r}{r+s} W_{1, n}^{*}(s), \quad n \geqslant 1 .
\end{aligned}
$$

Substituting (37) into (44) and (48), we get

$$
\begin{aligned}
& W_{0,0}^{*}(s)=\frac{\eta(\mu+\alpha)}{(\eta+s)(\mu+\alpha+s)}, \\
& W_{2,0}^{*}(s)=\frac{r(\mu+\alpha)}{(r+s)(\mu+\alpha+s)} .
\end{aligned}
$$

Substituting (37) and (50) into (46), we have

$$
W_{1,1}^{*}(s)=A \frac{\mu+\alpha}{\mu+\alpha+s}+B .
$$

Let $n:=n-1$ in (45) and substitute it into (47), we have

$$
W_{1, n}^{*}(s)=A^{n-1} W_{1,1}^{*}(s)+\frac{B\left(1-A^{n-1}\right)}{1-A}, \quad n \geqslant 2 .
$$


Substituting (52) into (53), we can obtain

$$
W_{1, n}^{*}(s)=A^{n} \frac{\mu+\alpha}{\mu+\alpha+s}+\frac{B\left(1-A^{n}\right)}{1-A}, \quad n \geqslant 1 .
$$

Substituting (54) into (45) and (49), respectively, and from (50) and (51), we get

$$
\begin{array}{ll}
W_{0, n}^{*}(s)=\frac{\eta}{\eta+s}\left(A^{n} \frac{\mu+\alpha}{\mu+\alpha+s}+\frac{B\left(1-A^{n}\right)}{1-A}\right), & n \geqslant 0, \\
W_{2, n}^{*}(s)=\frac{r}{r+s}\left(A^{n} \frac{\mu+\alpha}{\mu+\alpha+s}+\frac{B\left(1-A^{n}\right)}{1-A}\right), & n \geqslant 0 .
\end{array}
$$

Hence, the LST of the stationary sojourn time distribution of an arbitrary customer can be written as

$$
W^{*}(s)=\pi_{3,0} W_{3,0}^{*}(s)+\sum_{n=0}^{\infty} \pi_{0, n} W_{0, n}^{*}(s)+\sum_{n=1}^{\infty} \pi_{1, n} W_{1, n}^{*}(s)+\sum_{n=0}^{\infty} \pi_{2, n} W_{2, n}^{*}(s) .
$$

Substituting (37), (55), (54) and (56) into (57), (36) follows.

Then, the mean sojourn time is

$$
\begin{aligned}
E(W)= & -\left.\frac{\mathrm{d} W^{*}(s)}{\mathrm{d} s}\right|_{s=0} \\
= & \pi_{3,0} E\left(W_{3,0}\right)+\sum_{n=0}^{\infty} \pi_{0, n} E\left(W_{0, n}\right)+\sum_{n=1}^{\infty} \pi_{1, n} E\left(W_{1, n}\right)+\sum_{n=0}^{\infty} \pi_{2, n} E\left(W_{2, n}\right) \\
= & \frac{1}{\mu+\alpha} \pi_{3,0}+\sum_{n=0}^{\infty} \pi_{0, n}\left[\frac{1}{\eta}+\frac{C^{n}}{\mu+\alpha}+\frac{D\left(1-C^{n}\right)}{1-C}\right] \\
& +\sum_{n=1}^{\infty} \pi_{1, n}\left[\frac{C^{n}}{\mu+\alpha}+\frac{D\left(1-C^{n}\right)}{1-C}\right]+\sum_{n=0}^{\infty} \pi_{2, n}\left[\frac{1}{r}+\frac{C^{n}}{\mu+\alpha}+\frac{D\left(1-C^{n}\right)}{1-C}\right],
\end{aligned}
$$

where $C=\frac{\mu}{\mu+\alpha}, D=\frac{\eta+\mu q}{\eta(\mu+\alpha)}$.

\section{Numerical Results}

In this section, some numerical examples are presented to show the effect of the parameter on the mean system length.

In Figure 1 , let $\lambda=1, \eta=1.25, r=1.3, q=0.5, p=0.5$. It shows the impact of service rate $\mu$ on the mean system length $E[L]$ for different values of $\alpha$. As we can see from the graph, the mean system length $E[L]$ decreases with the increasing of $\mu$. When $\mu$ is fixed, the bigger $\alpha$ is, the smaller $E[L]$ is. Further, comparing these three curves, we find that when $\alpha$ becomes bigger, the slope of curve increases, i.e., the impact of service rate $\mu$ on $E[L]$ weakens. Obviously, it fits the fact that even if the customers are served at a low rate, once the disaster occurs, it will compel all customers present to leave. 


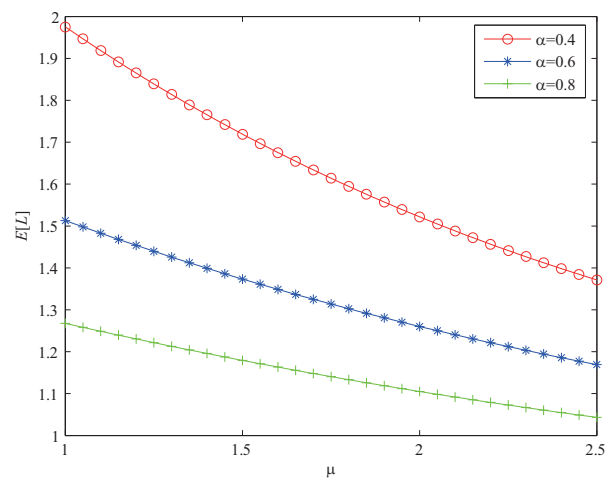

Figure 1 Effects of $\mu$ and $\alpha$ on the mean system length $E[L]$

In Figure 2, let $\lambda=1, \mu=1.5, \eta=1.25, q=0.5, p=0.5$. It shows the impact of $r$ on the mean system length $E[L]$ for different values of $\alpha$. As can be seen from the graph, the mean system length $E[L]$ decreases as $r$ increases. When $r$ is fixed, $E[L]$ becomes smaller as $\alpha$ becomes bigger. Further, comparing these three curves, we can get that when $\alpha$ becomes bigger, the slope of decreases, i.e., the effect of repair rate $r$ on $E[L]$ strengthens. This is because the more frequently the disasters occur, the higher rate $r$ the system needs to repair it.

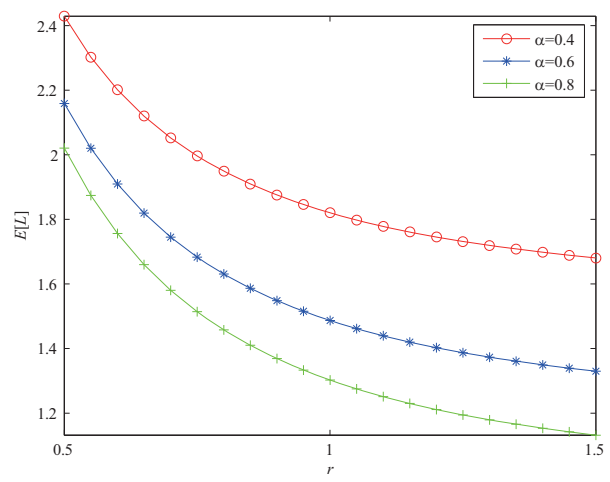

Figure 2 Effects of $r$ and $\alpha$ on the mean system length $E[L]$

In Figure 3, let $\lambda=1, \mu=1.5, \alpha=0.6, r=1.3$. It shows the influence of the probability of taking a vacation $q$ on the mean system length $E[L]$ for different values of $\eta$. As is shown in the graph, the mean system length $E[L]$ increases with the increase of the probability of taking a vacation $q$. When $\eta$ is fixed, $E[L]$ becomes bigger as $q$ becomes bigger. Further, we notice that when $q=0$, the queue model reduces to an $\mathrm{M} / \mathrm{M} / 1$ queue with disasters and no vacation. Therefore, the rate $\eta$ has no effect on $E[L]$. On the contrary, when $q=1$, the queue model translates to an $\mathrm{M} / \mathrm{M} / 1$ queue with disasters and single vacation. Thus $E[L]$ decreases with the increase of $\eta$. Comparing these three curves, we notice that as $q$ increases or $\eta$ decreases, the mean system length $E[L]$ becomes larger. It is obvious that taking more or longer vacations takes the server more time, and makes the waiting line longer. 


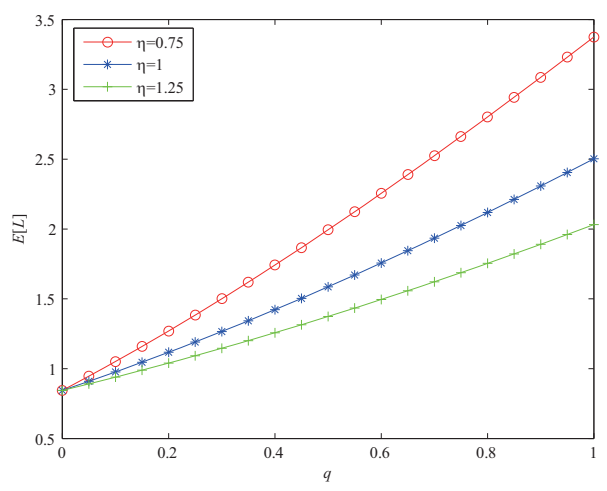

Figure 3 Effects of $q$ and $\eta$ on the mean system length $E[L]$

\section{Conclusion}

This paper studied a single-sever queue with disasters and repairs under Bernoulli vacation schedule. We obtained the PGF for the number of customers in the system under stationary by solving the balance equations. Some performance measures such as the mean system length, the probability of the server in different states, the rate at which disasters occur and the rate of initiations of busy period are presented. Further, we derived the LST of the sojourn time distribution and obtained the mean sojourn time. Finally, we presented some numerical examples to show the influence curves of parameters on the mean system length. We expect that the results can be applied to more practical queueing systems.

Acknowledgements The authors gratefully acknowledge the editor and two anonymous referees for their insightful comments and helpful suggestions that led to a marked improvement of the article.

\section{References}

[1] Kumar B K, Arivudainambi D. Transient solution of an M/M/1 queue with catastrophes. Computers \& Mathematics with Applications, 2000, 40(10): 1233-1240.

[2] Yang W S, Kim J D, Chae K C. Analysis of M/G/1 stochastic clearing systems. Stochastic Analysis and Applications, 2002, 20: 1083-1100.

[3] Economou A, Fakinos D. A continuous-time Markov chain under the influence of a regulating point process and applications in stochastic models with catastrophes. European Journal of Operational Research, 2003, 149(3): 625-640.

[4] Gani J, Swift R J. Death and birth-death and immigration processes with catastrophes. Journal of Statistical Theory and Practice, 2007, 1(1): 39-48.

[5] Shin Y W. Multi-server retrial queue with negative customers and disasters. Queueing Systems, 2007, 55(4): 223-237.

[6] Yechiali U. Queues with system disasters and impatient customers when system is down. Queueing Systems, 2007, 56(3-4): 195-202.

[7] Jolai F, Asadzadeh S M, Taghizadeh M R. Performance estimation of an email contact center by a finite source discrete time Geo/Geo/1 queue with disasters. Computers \& Industrial Engineering, 2008, 55(3): 543-556.

[8] Park H M, Yang W S, Chae K C. Analysis of the GI/Geo/1 queue with disasters. Stochastic Analysis and Applications, 2009, 28(1): 44-53. 
[9] Boudali O, Economou A. Optimal and equilibrium balking strategies in the single server Markovian queue with catastrophes. European Journal of Operational Research, 2012, 218(3): 708-715.

[10] Boudali O, Economou A. The effect of catastrophes on the strategic customer behavior in queueing systems. Naval Research Logistics, 2013, 60(7): 571-587.

[11] Sudhesh R. Transient analysis of a queue with system disasters and customer impatience. Queueing Systems, 2010, 66(1): 95-105.

[12] Dimou S, Economou A. The single server queue with catastrophes and geometric reneging. Methodology and Computing in Applied Probability, 2013, 15(3): 595-621.

[13] Kim B K, Lee D H. The M/G/1 queue with disasters and working breakdowns. Applied Mathematical Modelling, 2014, 38(5): 1788-1798.

[14] Yi X W, Kim J D, Choi D W, et al. The Geo/G/1 queue with disasters and multiple working vacations. Stochastic Models, 2007, 23(4): 537-549.

[15] Economou A, Gómez-Corral A. The batch Markovian arrival process subject to renewal generated geometric catastrophes. Stochastic Models, 2007, 23(2): 211-233.

[16] Mytalas G C, Zazanis M A. An $\mathrm{M}^{X} / \mathrm{G} / 1$ queueing system with disasters and repairs under a multiple adapted vacation policy. Naval Research Logistics, 2015, 62(3): 171-189.

[17] Jiang T, Liu L, Li J. Analysis of the M/G/1 queue in multi-phase random environment with disasters. Journal of Mathematical Analysis and Applications, 2015, 430: 857-873.

[18] Jiang T, Liu L. The GI/M/1 queue in a multi-phase service environment with disasters and working breakdowns. International Journal of Computer Mathematics, 2015, DOI: 10.1080/00207160.2015.1128531.

[19] Ke J C, Wu C H, Zhang Z G. Recent developments in vacation queueing models: A short survey. International Journal of Operations Research, 2010, 7(4): 3-8.

[20] Zhang X, Liu L, Jiang T. Analysis of an M/G/1 Stochastic Clearing Queue in a 3-Phase Environment. Journal of Systems Science and Information, 2015, 3(4): 374-384. 